\title{
Method of Device Diagnostics, Operating Status LOGgING AND CONFIGURATION CHANGES
}

\author{
Marta Blahova, Vaclav Mach, Ondrej Zimek \& Milan Adamek
}
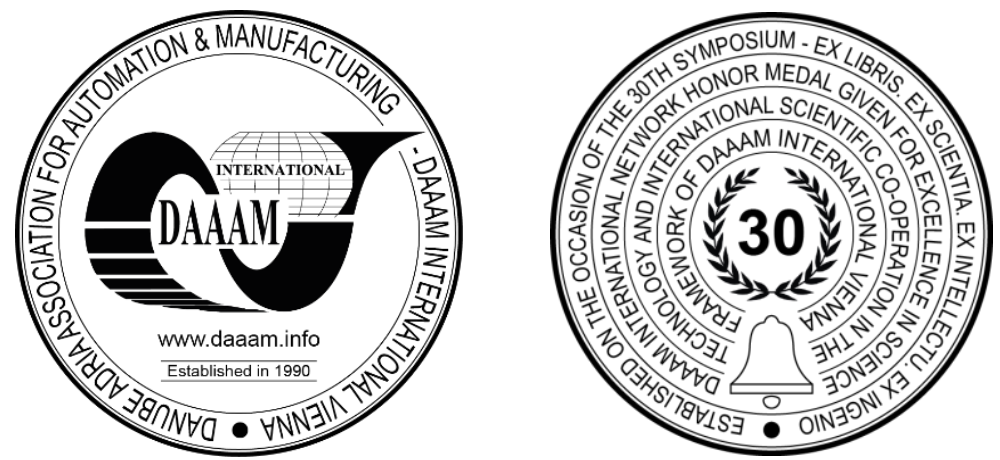

This Publication has to be referred as: Blahova, M[arta]; Mach, V[aclav]; Zimek, O[ndrej] \& Adamek, M[ilan] (2019). Method of Device Diagnostics, Operating Status Logging and Configuration Changes, Proceedings of the 30th DAAAM International Symposium, pp.1014-1018, B. Katalinic (Ed.), Published by DAAAM International, ISBN 978-3-90273422-8, ISSN 1726-9679, Vienna, Austria

DOI: $10.2507 / 30$ th.daaam.proceedings.141

\begin{abstract}
The aim of this research is to design a suitable design for a diagnostic device that will periodically monitor and record selected quantities in the device. The diagnostic equipment must be as flexible as possible, as the design will be applicable to all output electronic equipment of this project. This means that the diagnostic design can be applied to all control units, add-on modules or turnstile controllers. Each checked element contains different quantities that are valid for correct diagnostics. Therefore, there is a desire for a uniform design that can be customized based on the device. The data itself should include the exact time and date the measurement occurred. This information should be kept for several years for back diagnosis. Furthermore, the data should be stored primarily on the device with the possibility to transfer it to the PC via the given interface. Baseline states should be available directly on the device for immediate status. This information will be displayed via a small integrated display directly on the PCB. The data itself should be stored on a medium that retains the information even when the power supply is interrupted. The diagnostic part itself should be equipped with a backup battery, which will be functional even after the failure of the primary power supply for possible failure diagnosis. The design should consist of a microcomputer that will record events that can be updated with firmware to accommodate the board's needs. A simple program that visualizes the data for easier diagnostics will be needed to view the recorded data more easily.
\end{abstract}

Keywords: Diagnostics of electronic devices; electrical equipment; control units; integrated circuit

\section{Diagnostics of electronic devices}

All technical equipment must operate in such a way as not to endanger the persons operating or merely using them and to avoid their accidents. For this reason, it is necessary to determine their current technical condition or their functional properties. Although many diagnostic methods have been developed and used for various devices, the effectiveness of these methods depends primarily on the capabilities of the workers using or evaluating the measurement results.

This statement is particularly important for electrical equipment that can easily endanger people's health or lives through poor performance, failure, or failure. The huge expansion of electrical equipment in industry, transport, households, etc., where they are often operated by unqualified persons, underlines this importance. For these reasons, electrical equipment is prescribed and inspected within the time limits given by the importance and magnitude of the risk that each type of 
equipment presents. Inspection activities are carried out by inspection technicians, who must pass very strict tests at the technical supervision offices.

However, diagnostic work is carried out by workers with electrical education, but without any verification of their expertise in the diagnostic method used or its suitability for the case. In electrical engineering, the diagnostician must have extraordinary expertise in the structure of electrical materials, their technological processing, aging and degradation, principles of functional properties of electrical equipment, principles of destructive and non-destructive tests, etc. For this reason, a qualification and certification program must be created. It laid down the basic conditions to be met by an electrodiagnostic worker in order to ensure that his conclusions are realistic and plausible.

This work was prepared to determine qualification and certification of persons whose specific work requires adequate knowledge of principles of electrical engineering, their practical applications in electrical machines, electrical power distribution, in safe work with electrical equipment, etc. in electro-diagnostics.

\subsection{Online Diagnostics}

To test the condition of electrical equipment, we have diagnostic methods, both general and targeted to the specific possible failure. From the point of view of the diagnostic system, functional diagnostics is clearly preferable, which uses measurable quantities generated by the device itself during its normal operation, as opposed to test diagnostics, where the test quantity is generated by a diagnostic device and usually requires machine shutdown and preparation for measurement.

By not disturbing the normal operation of the machine, functional diagnostics can be performed continuously and can be part of monitoring and on-line systems. The trend in the field of machine diagnostics is directed to the use of these methods to create an online system. Combining the measuring instrument and the evaluation part into one unit today, usually using computer technology and virtual instrumentation methods, allows for more complex evaluation of continuously measured diagnostic quantities to use an expert system and allow more complex evaluation than just monitoring whether any of the parameters deviates from predetermined limits.

\subsection{Diagnostics of control units}

When interconnecting multiple integrated circuits, there is a problem that may cause incorrect soldering of the IO pins to the PCB. A problem may also occur when soldering other components that are directly connected to the IC. Usually it is incorrect soldering or multi-way fitting together. Using the Boundary Scan (BS) extension, these pins can be used to control the individual pins of the circuit via serial communication and also to read the current states on its pins. One such program is described in the next chapter. The general wiring diagram is shown in the following figure.

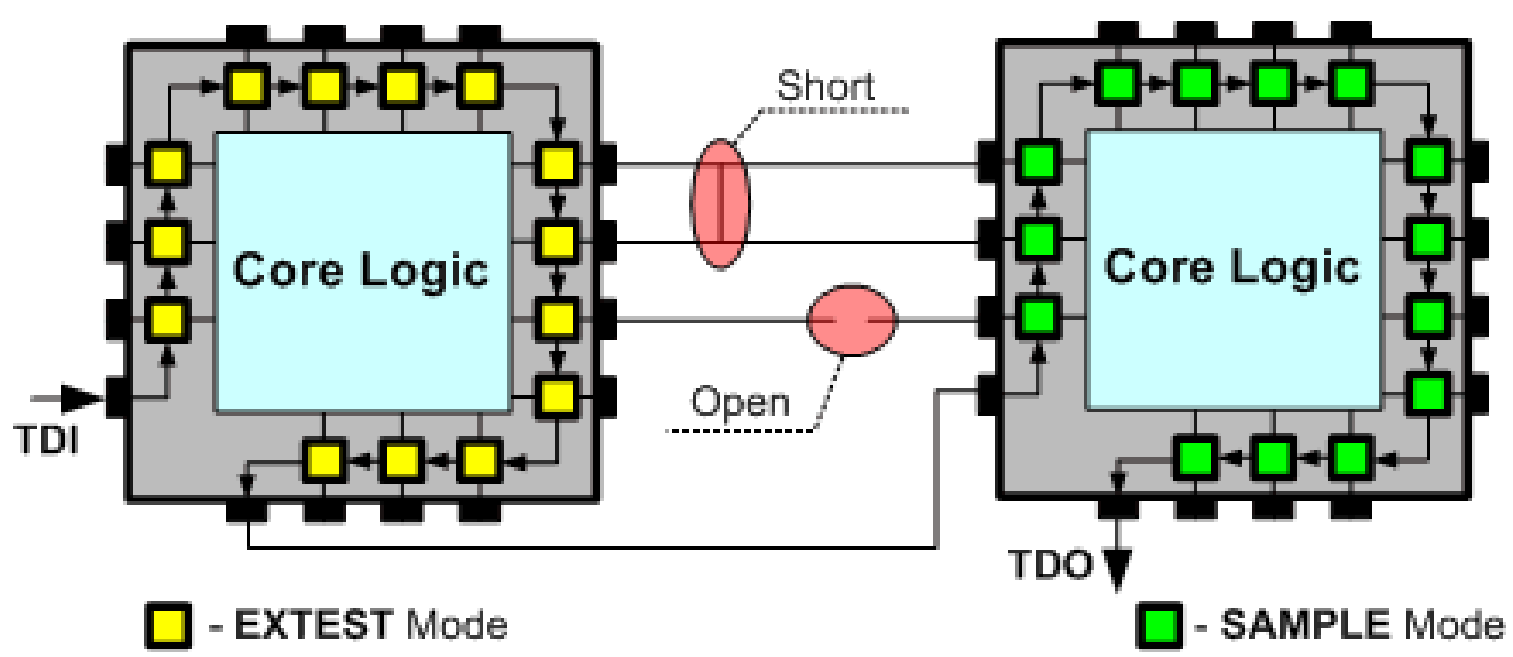

Fig. 1 - Scheme of Boundary Scan [3]

Specifically, it is a superstructure that links the individual pins of the core with its physical shell. Each LUN contains input and output pins. With the BS extension, the pins from the logic unit are first inserted into the Boundary Scan Cell and only then to the physical pins that lead from the package. The external program can then disconnect the LUN and send signals to the output pins. In practice, several ICs are connected in series to one PCB. If all have a JTAG body, BS diagnostics and testing can be applied to this chain. 
The superstructure consists of several basic functional units. Thanks to its more complex structure, the superstructure can be compared to a simple controller that can handle simple instructions and also work with serial data strings. Each superstructure contains an internal memory used to store both instructions and the data itself. In practice, the data is first loaded into the memory, then the instruction and the control signal output the corresponding instruction. Each IO has several special pins, which serve as input, output and control pins of the body. These are:

- Boundary Scan Cell

- Test Access Port

- By-pass register

Boundary Scan Cell - This is the cell that connects the superstructure and the physical pins of the integrated circuit. In normal operation, individual cells only transmit a signal from the logic unit to the output of the integrated circuit. When the superstructure is activated, the cells ignore the signals from the LUN.

Test Access Port - Test Access Port is the main element of the whole body. It is responsible for all operations in the superstructure and also generates an internal clock that controls all internal elements. Usually, 4 signals are input to the TAP. These signals control the input and output data. TAP can also record and store the necessary instructions and data in its internal memory. [4]

By-pass register - The more integrated circuits in one chain, the slower the transfer of information from one cell to another. During testing, the individual pins can be easily skipped. The by-pass register contributes to this feature. Depending on the current setting, the register can skip individual ICs, or even the entire circuit if required. The following figure shows the arrangement of individual by-pass register cells.

\subsection{Joint Test Action Group}

The Joint Test Action Group (JTAG) is a standard that was created in 1985 for the development and testing of PCBs. In 1990, this standard was adopted as IEEE 1149.1-1990. It is a chip implementation as a complementary tool for digital communication. [5] This is a serial port that can be used to transmit both instructions and data. An external device is connected to the test port to test the device. Additional pins are added to the chip that has a JTAG interface. The number of these pins may vary depending on the version. The basic pins include the following:

- $\quad$ Test Data In (TDI)

- Test Data Out (TDO)

- TCK (Test Clock)

- Test Mode Select (TMS)

Test Data In (TDI) - Two types of signals can appear at the TDI input. The first signal can be instructions that are sent directly to the instruction register and the second signal the test data itself can be stored in the data register. The TDI reacts to the falling edge of the clock signal. [4]

Test Data Out (TDO) - This output receives data from the instruction register or test data register. With the administrative instruction and the falling edge, the data is sent to the next chip, which is located in the string. If data must be stored in the registry, the TDO is switched to an inactive state. [4]

Test Clock Input (TCK) - The clock frequency is completely independent of the system clock. This means that the test chip may be in sync with others. The main emphasis is on the edges of the clock signal, where the leading edge is used to record TDI data to TAP and the falling edge is used to output TDO data. [4]

Test Mode Select (TMS) - The test process operation must be controlled by a specific sequence of commands. Individual states change at the falling edge of TCK. These sequences are fed to TAP where this information is used to test logic circuits. TMS is equipped with pull-up resistor to be able to respond to logic [4]

\section{Design of system for diagnostics}

The amount of data generated during preparation can only be estimated in advance. It is significantly dependent on the number of monitored operating parameters and the way they will be measured. Physical quantities such as temperature, which can be sampled with a period of about $1-2 \mathrm{~Hz}$. Conversely, recording machine vibration along with a position for subsequent diagnostic analysis requires much faster sampling. Considering the sampling rate of $5 \mathrm{kHz}$ and the size of one sample $2 \mathrm{~B}$, the data rate is $10 \mathrm{kB} / \mathrm{s}$. If the instantaneous turnstile position of both 8 -bit compression units of the given type is stored simultaneously, the data flow will increase to $20 \mathrm{kB} / \mathrm{s}$. 
Permanent operation, for which the device is to be destined, does not allow to use such EEPROM or SPI Flash memory for storing such large data flows because of insufficient capacity (tens of kB or MB units). For sufficient user comfort, the storage medium should hold a recording for at least 1 year. Its capacity must be at least 2 GB. You can use USB Flash Disk or SD memory card. Both types of storage media meet both capacity and speed requirements. The advantage is also low price per unit of storage capacity. Alternatively, it is possible to implement the function of data logging at the other end of the communication line, ie on the service PC. This option would be effective especially if a dedicated PC would serve as a server. [7]

\subsection{Concept and design}

In order for the datalogger to fulfill the specified functions, a circuit design and design was created. In this chapter the concept and circuit design are described with focus on the selection of individual components. The design is based on the requirements for simplicity of measurement of atmospheric quantities without the need for complicated sensor calibration, minimum consumption, battery charging and direct USB connection without the need for a converter and, last but not least, on the small size of the device.

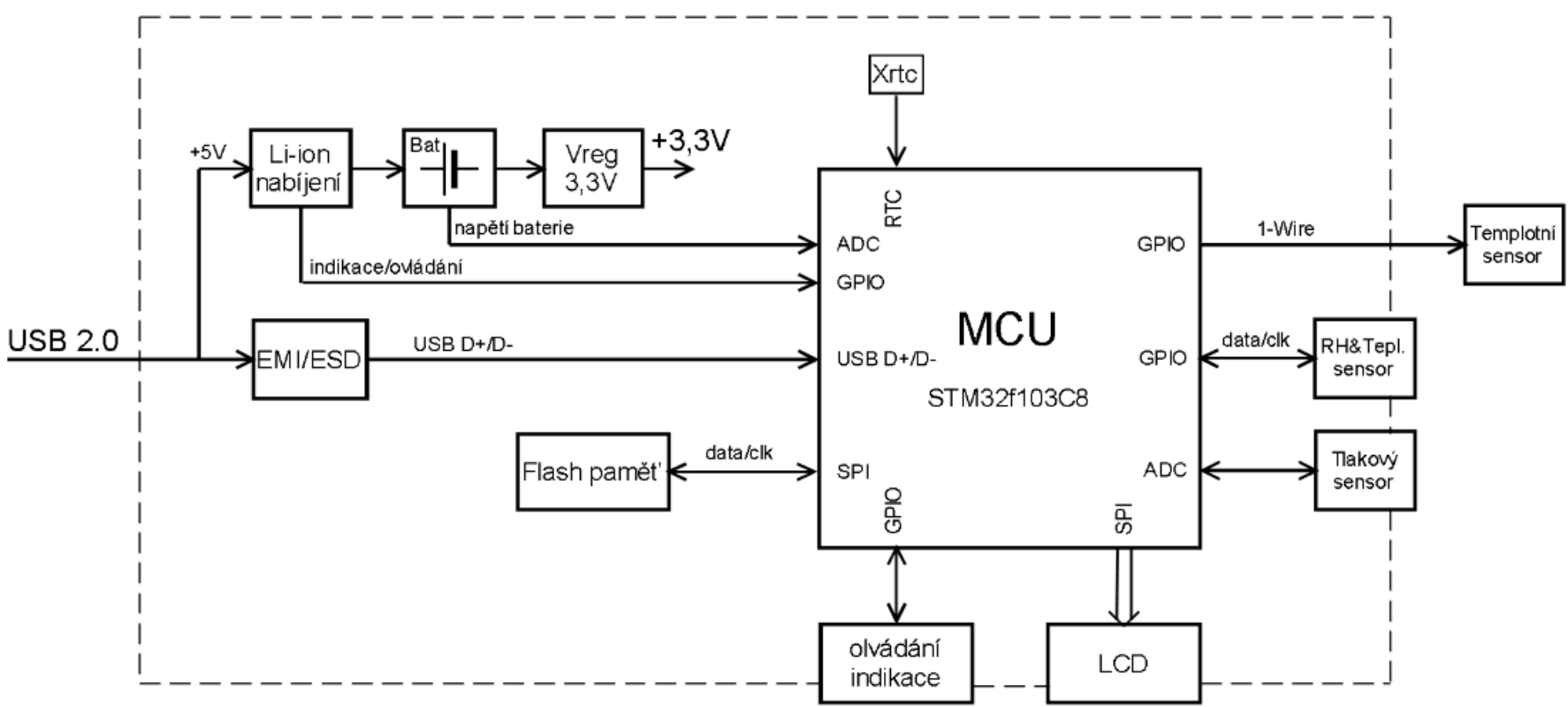

Fig. 2 - Block diagram of datalogger [7]

All operations are managed and controlled by the MCU. It starts the measurement of atmospheric quantities by means of sensors, compresses and saves the acquired data into flash memory, or displays operating information on the LCD display. The power branch circuit consists of a charger, a Li-Ion battery and a voltage stabilizer. The MCU also monitors the battery status and controls the charging process when the datalogger is connected to a USB line and provides data transfer to the PC.

The STH15 sensor was selected for relative humidity measurement. It consists of a band gap sensor for measuring temperature and a capacitive sensor for measuring relative humidity, an A / D converter and a signal processing unit. Measured humidity and temperature values are calibrated and converted to digital values. The sensor operates in an environment where condensation does not occur, but from the recorded values the dew point temperature can be calculated. The sensor has a two-wire serial interface for data acquisition. To obtain real values of measured values, the data obtained from the sensor must be converted using the following relations.

The MP3H6115A sensor was selected for atmospheric pressure measurement. It is an absolute pressure sensor. The core of the sensor is a semiconductor resistive measuring element with temperature compensation and an output amplifier. The sensor output is analog, the output voltage is applied to the A / D converter MCU.

The DS18S20 sensor [5] was selected for remote temperature measurement. The measured temperature is converted to a digital value by the sensor and stored in the internal memory. The sensor has 1 -wire interface, for connection to MCU is used one data wire and ground wire. It allows the "parasitic" mode of powering the sensor. In this mode, the sensor is fed through the data line using a strong pull-up resistor. When performing more demanding sensor operations, such as temperature conversion or internal memory operations, the data line is bound by a MOSFET transistor directly to power the data logger.

MCU STM32f103 was chosen as the datalogger control unit. It is a 32-bit ARM processor with Cortex-M3 core. It was chosen for the device because it allows direct connection to a PC via a full-speed USB 2.0 bus. It has an RTC unit, which is needed for time records when logging measured data. One of several low power modes can be used. An SPI bus is available to connect the selected memory and LCD display. 
A sufficiently large non-volatile memory is required to store the measured data. The AT25DF161 flash memory has been selected. The memory is connected to the control unit via the SPI serial interface. The memory provides sufficient capacity of $16 \mathrm{Mb}$.

The EADOGM132E graphic display has been selected to display the most recently recorded data and status information of the datalogger. The display resolution is $132 \times 32$ dots, it operates with a uniform $3.3 \mathrm{~V}$ power supply and low consumption of $140 \mu \mathrm{A}$ in the backlight mode.

A rechargeable Li-Ion battery has been selected to power the datalogger. The battery voltage is measured by the A / $\mathrm{D}$ converter MCU. At the A / D converter input, the battery voltage is adjusted by a divider with a split ratio of 0.5 . From the measured voltage, the approximate remaining battery capacity can be determined by the battery discharge characteristic. The battery is charged directly from the USB port of the PC. The integrated charger NCP1835B with CCCV charging profile has been selected for this purpose.

\section{Conclusion}

The result of the research is the design of a diagnostic device for recording and storing the necessary data, variables and events for back-checking or diagnostics. The diagnostic device itself is located on the printed circuit boards that perform the function. For this reason, the diagnostic device is very flexible and contains many inputs for sensing various quantities that can be processed by the microcomputer used. The microcomputer is connected to an SD card, which has sufficient data space for recording, but also the possibility to remove or replace the card. The design itself also includes a USB connector, which can be used to connect the device to a PC and then use the program to manage.

For instant access to up-to-date information, a diagnostic design also includes a small LCD to display basic information. This function can be used without connecting the diagnostics to the PC. The basic diagnostic design must be modified before application. The connection design can be easily added to the circuit diagram of the board using the function. Basic sensors are located directly in the design and to add external sensors it is necessary to complete the design with connectors for their connection.

The created device is able to perform all the functions given by the work with some extensions. The basic extensions are added pressure sensor and external temperature sensor, more logging options, but also measurement of atmospheric quantities outside the logging intervals and displaying their values on the display or their direct transfer to the PC and display in the control application. The created datalogger has the potential for further development, which mainly concerns operation without the need to connect the datalogger to the PC, signaling of possible faults of sensors and other components. It is also possible to improve the compression system of the stored data and achieve a higher compression ratio.

\section{Acknowledgments}

This research was based on the support of the Internal Grant Agency of Tomas Bata University in Zlín, the IGA / FAI / 2019/003, IGA/Cebia Tech/2019/003 project and the Institute of Safety Engineering, Faculty of Applied Informatics.

\section{References}

[1] Chou, Y. \& Yao, L. (2009) Automatic Diagnostic System of Electrical Equipment Using Infrared Thermography. International Conference of Soft Computing and Pattern Recognition pp. 155-160. DOI: 10.1109/SoCPaR.2009.41. ISBN 978-1-4244-5330-6.

[2] Bernat, P. (2015) On-Line Diagnostics Of Electrical Torque Machines. Available from: http://homen.vsb.cz/ ber30/texty/diagnostika/DIAGO98.pdf

[3] JTAG Toolkit. JTAG Tools Source Forge, 2014. Available from: http://jtagtools.sourceforge.net/images/jtg5.gif

[4] Maunder, C. \& Tulloss, R. (1991) The test access port and boundary-scan architecture. Alamitos, Calif. Computer Society Press, c1991 ISBN 08-186-60708.

[5] IEEE Standard for Reduced-Pin and Enhanced-Functionality Test Access Port and Boundary-Scan Architecture The official IEEE 1149.7 Standard

[6] EEVblog (2018) What is JTAG and Boundary Scan. Available at: https://www.youtube.com/watch?v=TlWlLeC5BUs. EEVblog's channel

[7] Romanek, K. (2011) Independent Datalogger with USB Connection. Brno, Brno University of Technology, Faculty of Electrical Engineering and Communication Technologies.

[8] Novobilsky, P. (2011) Independent Datalogger With USB Connection. Brno University of Technology, Faculty of Electrical Engineering and Communication Technologies.

[9] Hubálek, J. \& Adámek, M. (2001) Microsensors and Microelectromechanical Systems. Brno: Brno University of Technology. 